\title{
MULTI-OBJECTIVE STRATEGY FOR OPTIMIZING REPETITIVE CONSTRUCTION PROJECTS USING LINEAR PROGRAMMING MODELS
}

\author{
Sherif M. Hafez ${ }^{1}$, Enas S. Morgan², Mohamed A. Hamad² \\ ${ }^{1}$ Professor of Construction Engineering, Department of Civil Engineering, Alexandria \\ University, Egypt. \\ E-mail: sherif.hafez@alexu.edu.eg \\ ${ }^{2}$ M.Sc. Candidates, Department of Civil Engineering, Alexandria University, Egypt. \\ E-mail: enas_morgan@yahoo.com,m_fadeel@hotmail.com
}

\section{ABSTRACT}

Decision making has become much more complicated than in the past due to increased decision alternatives, uncertainty, and cost of making errors. As a result, it is very difficult to rely on a trial and error approach in decision making. Nowadays business managers are dealing with different types of projects ranging from implementing a large scale manufacturing plant to a simple sales campaign. While dealing with projects, to become competitive, sometimes it is required to complete a project within the predetermined deadline to keep cost at lowest possible level. Failure to do so ultimately leads to increase in total cost. This would direct managers to encounter a decision situation: which activities of the project will be crashed to minimize the total cost of crashing project. In this paper, we provide a hypothetical example to clarify the framework of how to convert from LOB to CPM and then how to create a model to crash a project time to reach an optimum time-cost solution. Microsoft Excel custom made sheets used to the conversion, also Solver add-in used to solve the model while it implements Linear Programming. As a check, results from Solver and LiPS software are compared.

Keywords: Line-Of-Balance, LOB, Linear Programming, LP, Project Crashing, Time-Cost Trade-Off, CPM

\section{INTRODUCTION}

Most of construction managers are continually facing a situation in which they must take a decision whether to complete the project sooner than originally specified in the contract because of the clients request and /or to optimize the cost of expediting.

The planned duration is decreased by crashing all critical activities either by authorizing overtime work or applying additional resources.

\section{EXISTING PROJECT-PLANNING TECHNIQUES}

The technique used for project scheduling will vary depending on the project's size, complexity, duration, personnel, and owner's requirements.

The project manager must choose a scheduling technique that is simple to be used and is easily interpreted by all project participants, Oberlender, G. (2008). 


\subsection{Bar Chart}

\subsection{Network-Based Methods.}

There are two widely known network based techniques:

3.2.1. Critical Path Method, "CPM"

3.2.2. Program Evaluation and Review Technique, "PERT"

\subsection{Linear Projects}

Several techniques were developed for projects with discrete units, such as floors, houses, offices, etc. The names used have included the following:

1. Line Of Balance, "LOB" [O'Brien, J. (1969)];

2. Construction planning techniques [Peer, S. (1974)];

3. Vertical Production Method, "VPM" [O'Brien, J. (1975)];

4. Time space scheduling method [Stradal, O. (1982)];

5. Time-location matrix model [Carr, $R$. (1993)].

Line-Of-Balance, "LOB" is one production scheduling and controlling technique, which tries to surpass the CPM difficulties for the multi-story building scheduling. It was developed into manufacturing environment by the US Navy, and had its origins at the time of the World War II, Burke, R. (1996).

Hafez, S. (1997a), developed a tool for time and resource scheduling for repetitive construction projects, this has been done in three stages. This model called Modified Repetitive Project Model, "MRPM", which depends on the integration between the principles of line of balance method and critical path method. Crashing the critical activity can alter the direction of the critical path. Therefore, it is necessary to re-examine the critical path after each cycle of applying the developed system, to ensure that the overall project duration is shortened to the required limit. Otherwise, the current critical path will be targeted for further crashing.

\subsection{Non-Linear Projects}

Russell, A. (1993) commented that the majority of construction projects with repetitive activities are non-linear.

Hafez, S. (2005), surveyed the different issues, which related to schedule repetitive construction process. It can be used in the development of a computerized scheduling system. Firstly, applying resource-driven scheduling methods, visual presentation of line of balance diagram, optimize project cost, and resource utilization were discussed. Finally, it studied the acceleration routine, and integration scheduling methods.

\section{LINEAR PROGRAMMING TECHNIQUE}

Linear programming is a tool for decision making under certain situation. So, the basic assumption of this approach is that we have to know some relevant data with certainty. The basic data requirements are as follows:

1. The project network with activity time, which can be achieved from PERT and CPM.

2. To what extent an activity can be crashed.

3 . The crashing cost associated with per unit of time for all activities.

4. The same data mentioned in 2 and 3 for each activity-option if available.

To reduce the time to complete the activity, more resources are applied in the form of additional personnel and overtime. As more resources are applied, the duration is shortened, but the cost rises. The maximum effort is applied so that the activity can be completed in the shortest possible time. 


\subsection{Literature Review}

Selinger (1980) developed a dynamic programming model of a linear project. His work ignored to incorporate the cost as decision variable in the optimization process. As extension of the Selinger's work, Russel and Caselton (1988) formalized a N-stage dynamic programming solution into two state variable to determine the minimum project duration. In the optimization process, the developed model ignored the activities costs as a decision variable. Reda (1990) developed a linear programming to identify minimum cost maintaining constant production rates and repetitive projects. This method can only be used for non-typical linear project and not applicable to construction projects. Most of the developed models assume the activities are accomplished serially. In reality, most construction activities are accomplished concurrently while others accomplished serially.

Elmaghraby (1997) considered completion schedules on an arbitrary set of milestone events by developing an efficient algorithm to determine the project schedule, which minimizes the sum of the total cost plus penalties for late completion. Another extension was by Moore (1998) by using goal programming to consider multiple objectives, such as completion times, resources leveling and operation within a limited budget. Senouci (1996) presented a dynamic programming formulation for the scheduling of non-sequential or non-serial activities to determine the project time-cost profile which determines possible project duration and their minimum project total cost. The formulation considers the effects of interruptions, minimum project direct cost, and minimum project duration.

\section{RESEARCH OBJECTIVES}

This research study is expected to transform the optimization of repetitive construction resource utilization problems from an intractable problem to a feasible and practical one. The application of these research developments in planning the construction projects holds a strong promise to:

1. Increase the efficiency of resources used for typical-repetitive large-scale construction projects;

2. Reduce construction duration period;

3. Minimize construction cost (sum of direct cost and indirect cost).

\subsection{Considered Assumptions}

The mathematical formulation of the present model is based on the following assumptions:

1. No idle time is allowed for employed crews,

2. A constant average duration is set for the same activity at all stages to maintain a constant production rate.

3. The learning phenomenon, is neglected;

4. The work on each activity is conducted by one unit at a time.

\subsection{Employed Techniques}

1. For each activity $(\mathrm{k})$, (where $\mathrm{k}=1,2, \ldots, \mathrm{K})$ in the typical-repetitive network, $\mathbf{L O B}$ is used to represent the activity schedule at all stages in project time plan;

2. Transformation from the traditional LOB to modified CPM must be done in the model; 
3. Each activity $(\mathrm{k})$, (where $\mathrm{k}=1,2, \ldots, \mathrm{K})$ has a time buffer $\left(\mathrm{TB}_{\mathrm{k}, \mathrm{kk}}\right)$, at each stage $(\mathrm{s})$, (where $\mathrm{s}=1,2, \ldots, \mathrm{S}$ ) between the completion time of the activity (k)and the start time of each following activity (kk) in the network;

4. Any two sequential activities may have a stage buffer $\left(\mathrm{SB}_{\mathrm{k}, \mathrm{kk}}\right)$, of a specific number of stages at any time to meet practical and / or technological purposes, this stage buffer has to be identified by the planner for these activities;

\section{FORMULATION OF THE PROPOSED MODEL}

\subsection{Objective function of Normal Duration and Cost (Optimum}

\section{Alternative):}

In this model, project duration will be estimate using a converted LOB to a new modified CPM for scheduling typical-repetitive large-scale construction projects.

\subsubsection{Objective Function Of Normal Duration:}

$$
\begin{aligned}
& Z_{1}^{n}=\min \sum_{k=1}^{k=K K}\left(N T_{K}^{n}+\left(F S_{m}\right)_{K, K K}^{n}\right) \\
& N T_{K}=S \times N D_{K}^{n} \\
& N T_{K K}=S \times N D_{K K}^{n} \\
& \text { No.of Alt(Solutions) }=n=\text { No. of Alt } t_{k 1} x \text { No. of Alt } t_{k 2} X \ldots \ldots \ldots \ldots \text { No. of Alt } t_{K} \\
& \mathrm{~TB}_{\mathrm{K}, \mathrm{KK}}^{\mathrm{n}} \geq F S_{K_{0}, K K} \\
& \mathrm{~TB}_{\mathrm{K}, \mathrm{KK}}^{\mathrm{n}} \geq S S_{K, K K}-N D_{K}^{n} \\
& \mathrm{~TB}_{\mathrm{K}, \mathrm{KK}}^{\mathrm{n}} \geq F F_{K, K K}-N D_{K K}^{n} \\
& \mathrm{~TB}_{\mathrm{K}, \mathrm{KK}}^{\mathrm{n}} \geq S F_{K, K K}-\left(N D_{K}^{n}+N D_{K K}^{n}\right)
\end{aligned}
$$

\subsubsection{Case of Critical Stage at First floor:}

For any two sequential activities $(\mathrm{k})$ and $(\mathrm{kk})$, if $\mathrm{ND}_{\mathrm{K}}^{\mathrm{n}} \leq \mathrm{ND}_{\mathrm{KK}}^{\mathrm{n}}$ then critical stage is firststage, (see Figure 1). Modified finish to start at a new CPM between these two sequentialactivities $(\mathrm{k})$ and $(\mathrm{kk})$ can be calculated as shown in the next formulas:

$$
\begin{aligned}
& \left(F S_{K, K K}\right)_{m}^{n} \geq T B_{K, K K}+(1-S) \times N D_{K}^{n} \\
& \left(F S_{K_{\imath}, K K}\right)_{m}^{n} \geq\left(S B_{K_{\imath}, K K}-S\right) \times N D_{K}^{n}
\end{aligned}
$$

Take the max of equation from (9 to 10)

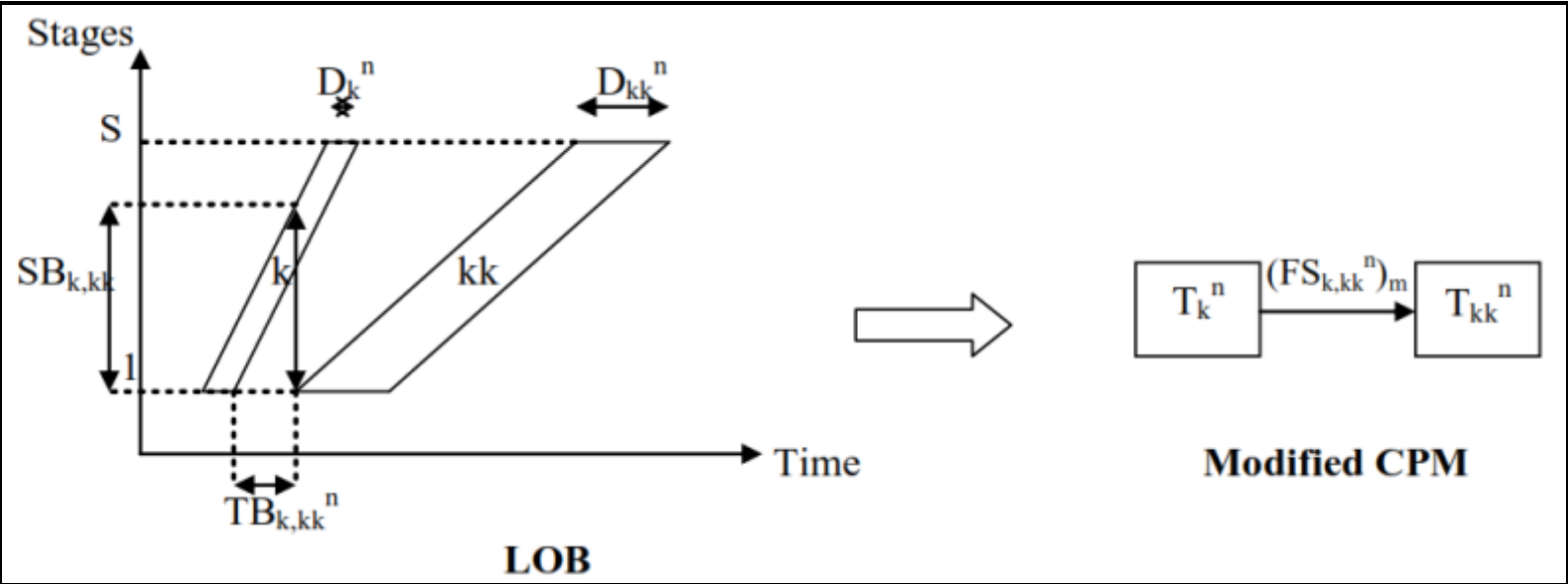

Figure 1: Modified CPM Integrated with LOB when Critical Stage is First Stage 


\subsubsection{Case of Critical Stage at Last floor:}

For any two sequential activities $(\mathrm{k})$ and $(\mathrm{kk})$, if $\mathrm{ND}_{\mathrm{K}}^{\mathrm{n}} \geq \mathrm{ND}_{\mathrm{KK}}^{\mathrm{n}}$, then critical stage is last stage, (see Figure 2). Modified finish to start at a new CPM between these two sequential activities $(\mathrm{k})$ and $(\mathrm{kk})$ can be calculated as shown in the next formulas:

$$
\begin{aligned}
& \left(F S_{K_{,}, K K}\right)_{m}^{n} \geq T B_{K_{,} K K}+(1-S) \times N D_{K K}^{n} \\
& \left(F S_{K_{0}, K K}\right)_{m}^{n} \geq\left(1+S B_{K_{0}, K K}-S\right) \times N D_{K K}^{n}-N D_{K}^{n} \\
& \text { Take the max of equation from (11 to 12) } \\
& \boldsymbol{P N D}_{\text {opt }_{\text {p }}}=\boldsymbol{Z}_{\mathbf{1}_{\min }^{n}}
\end{aligned}
$$

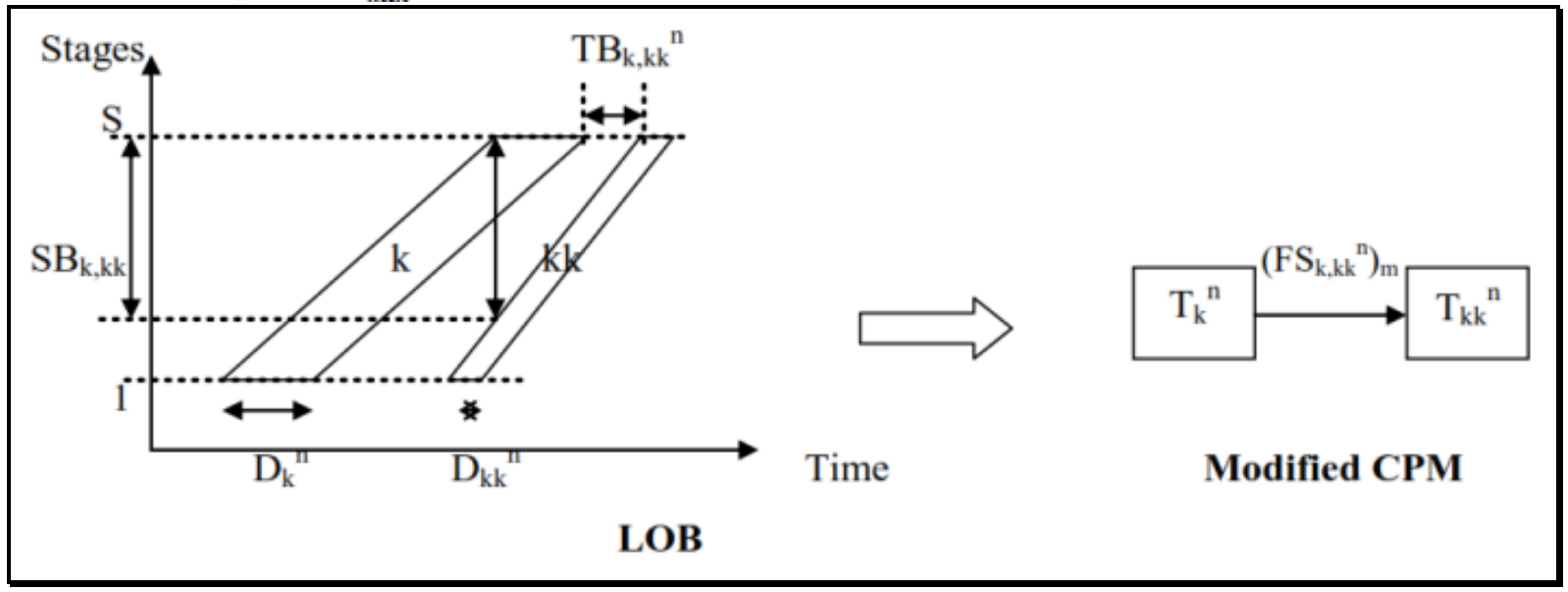

Figure 2: Modified CPM Integrated with LOB when Critical Stage is Last Stage Where:

- $\mathrm{Z}_{1}^{\mathrm{n}}=$ Minimum Normal Project Duration in (days) using resource utilization (n).

- $\mathrm{NT}_{\mathrm{K}}^{\mathrm{n}}=$ Normal Total durations in (days) at all stages of an activity (k) on the critical path at new CPM using resource utilization (n).

- $\left(\mathrm{FS}_{\mathrm{m}}\right)_{\mathrm{K}, \mathrm{KK}}^{\mathrm{n}}=$ Modified finish to start at a new CPM between two sequential activities using resource utilization (n).

- $\mathrm{S}=$ Number of stages in the project.

- $\mathrm{ND}_{\mathrm{K}}^{\mathrm{n}}=$ Normal Total durations in (days) at all stages of a predecessor activity ( $\mathrm{k}$ ) on the critical path at a new CPM using resource utilization (n)

- $\mathrm{ND}_{\mathrm{kk}}^{\mathrm{n}}=$ Normal Total durations in (days) at all stages of a successor activity (kk) on the critical path at a new CPM using resource utilization (n).

- $\mathrm{SB}_{\mathrm{K}, \mathrm{KK}}^{\mathrm{n}}=$ Stage buffer between the starts of two sequential activities (k) and (kk) at LOB.

- $\mathrm{TB}_{\mathrm{K}, \mathrm{KK}}^{\mathrm{n}}=$ Time buffer in (days) between two sequential activities finish (k) and start (kk) at LOB using resource utilization (n).

- $S T_{k}^{n}=$ Start time of a predecessor activity $(\mathrm{k})$ at first stage at LOB using resource utilization (n).

- $S S_{k, k k}=$ Start to Start in (days) between two sequential activities (k) and (kk) at LOB.

- $F F_{k, k k}=$ Finish to Finish in (days) between two sequential activities (k) and (kk) at LOB.

- $S F_{k, k k}=$ Start to Finish in (days) between two sequential activities (k) and (kk) at LOB.

Modified CPM can be constructed at a new CPM with modified finish to start between sequential activities in the network as shown in the previous formulas and then the time objective function with modified CPM can be conducted with Excel Spread sheet To Optimize The Minimum Project Normal Duration. 


\subsubsection{Objective Function Of Normal Cost:}

$Z_{2}^{n}=\operatorname{Min}\left(\left(S \times \sum_{k=1}^{k=k k} N C_{k}^{n}\right)+I C \times Z_{1}^{n}\right)$

- $Z_{2}^{n}=$ Min. Project Normal Cost $\{$ Direct $\}+\{$ Indirect $\}$ in (EGP) using resource utilization (n).

- $\mathrm{S}=$ Number of stages in the project

- $N C_{k}^{n}=$ Normal cost in (EGP) at one stage of an activity (k) at LOB using resource utilization (n)

- $\quad \mathrm{IC}=$ Daily indirect cost rate in (EGP/Day) along project life

The cost objective function as shown in the previous formulation (14) can be conducted with Excel Spread sheet To Optimize The Total Cost Of The Project

\subsubsection{Optimum Solution (Time-Cost-Trade- Off) for Normal Values}

$$
\begin{aligned}
& Z_{1}=\frac{P_{N D} O_{p t}-P N D_{\text {mean }}}{\delta_{N D}} \\
& Z_{2}=\frac{P N C_{O p t}-P N C_{\text {mean }}}{\delta_{N C}} \\
& \delta_{N D}=\frac{\sqrt{n \sum(P N D)^{2}-\left(\sum P N D\right)^{2}}}{n} \\
& \delta_{N C}=\frac{\sqrt{n \sum(P N C)^{2}-\left(\sum P N C\right)^{2}}}{n} \\
& Z_{\text {min }}=w_{1} \times Z_{1}+w_{2} \times Z_{2} \\
& w_{1}+w_{2}=1
\end{aligned}
$$

Where:

- $\mathrm{Z}=$ Time-Cost Objective Optimization Function.

- $\mathrm{w}_{1}=$ Weight of project duration focus.

- $\mathrm{w}_{2}=$ Weight of project cost focus.

- for optimum duration and corresponding optimum cost use $\left(\mathrm{w}_{1}=\mathrm{w}_{2}=0.50\right)$

\subsection{Crashing Project Duration Model:}

In this model, the optimum project normal duration obtained from the first model will be crashed using linear programming in order to solve the inequalities of the model.

\subsection{Objective Function:}

$$
\begin{aligned}
& \text { 6.3.1.1. Case of Min Project Total cost: } \\
& \mathrm{Z}=\frac{\min \sum_{K=1}^{N}\left(C S_{K} \times Y_{K}\right)+I C \times P D_{c}}{C S_{k}}=\frac{C C_{k}-N C_{k}}{N T_{k}-C T_{k}}
\end{aligned}
$$

\subsubsection{Case of Min Project Duration$$
\mathrm{Z}=\min P D_{c}
$$

\subsubsection{Constraints:}

\subsubsection{Non-negative constraints:}

All decision variables must be $\geq 0$ 


\subsubsection{Maximum reduction constraints:} $Y_{K} \leq \mathrm{NT}_{\mathrm{k}}-\mathrm{CT}_{\mathrm{k}}$

\subsubsection{Start Time Constraints:}

$S_{K K} \geq S_{K}+N D_{K}-Y_{K}+\left(F S_{m}\right) k, k k$

\subsubsection{Project duration constraint: $P D_{0} \leq P N D$ (opt)}

Where:

- $\mathrm{CS}_{\mathrm{k}}=$ Cost Slop of Activity $(\mathrm{K})$

- $\mathrm{CC}_{\mathrm{k}}=$ Crash Cost of Activity (K) in (EGP)

- $\mathrm{NC}_{\mathrm{k}}=$ Normal Cost of Activity (K) in (EGP)

- $\mathrm{NT}_{\mathrm{k}}=$ Normal Time in (days) of Activity (K) at all stages

- $\mathrm{CT}_{\mathrm{k}}=$ Crash Time in (days) of Activity (K) at all stages

- $\mathrm{S}_{\mathrm{k}}=$ Start Time of Activity $\mathrm{K}$ in (days)

- $\mathrm{Y}_{\mathrm{k}}=$ Amount of times in (days) that each activity $\mathrm{K}$ will be crashed

- PND (opt) = Optimum project duration obtained from formula No. (14)

- $\mathrm{PD}_{c}=$ Crashed Project Duration

\section{NUMERICAL EXAMPLE:}

A hypothetical 5 Activities network shown in figure (1) will serve as an example for demonstrating the proposed model. Table (1) shows the input data for the model. The studied project has 10 stages and the project Indirect Cost is 300 LE per unit time

Table 1: Activity Data for the Numerical Example

\begin{tabular}{|c|c|c|c|c|c|c|c|c|c|}
\hline $\begin{array}{l}\text { Activity } \\
\text { Name }\end{array}$ & $\begin{array}{c}\text { Depend } \\
\text { On }\end{array}$ & $\begin{array}{c}\text { Relation } \\
\text { Type }\end{array}$ & $\begin{array}{c}\text { Lag } \\
\text { Value }\end{array}$ & $\begin{array}{l}\text { Stage } \\
\text { Buffer }\end{array}$ & $\begin{array}{l}\text { Resource } \\
\text { Options }\end{array}$ & ND & CD & $\begin{array}{l}\text { NC per } \\
\text { all stages }\end{array}$ & $\begin{array}{c}\mathrm{CC} \\
\text { per all } \\
\text { stages }\end{array}$ \\
\hline \multirow{2}{*}{$\mathbf{A}$} & \multirow{2}{*}{-- } & \multirow{2}{*}{--} & \multirow{2}{*}{-- } & \multirow{2}{*}{--} & 1 & 20 & 17 & 40000 & 51000 \\
\hline & & & & & 2 & 23 & 22 & 50000 & 58000 \\
\hline \multirow{2}{*}{ B } & \multirow{2}{*}{ A } & \multirow{2}{*}{ SS } & \multirow{2}{*}{5} & \multirow{2}{*}{2} & 1 & 15 & 11 & 450000 & 485000 \\
\hline & & & & & 2 & 16 & 14 & 350000 & 371000 \\
\hline \multirow{2}{*}{ C } & \multirow{2}{*}{ A } & \multirow{2}{*}{ FS } & \multirow{2}{*}{2} & \multirow{2}{*}{1} & 1 & 13 & 12 & 200000 & 208000 \\
\hline & & & & & 2 & 26 & 24 & 150000 & 154000 \\
\hline \multirow{2}{*}{ D } & \multirow{2}{*}{ A } & \multirow{2}{*}{ SS } & \multirow{2}{*}{0} & \multirow{2}{*}{3} & 1 & 14 & 12 & 400000 & 406000 \\
\hline & & & & & 2 & 18 & 16 & 320000 & 325000 \\
\hline \multirow[t]{3}{*}{$\mathbf{E}$} & $\mathrm{B}$ & SF & 12 & 1 & 1 & 12 & 10 & 300000 & 309000 \\
\hline & $\mathrm{C}$ & FS & 0 & 2 & 2 & 21 & 19 & 240000 & 264000 \\
\hline & $\mathrm{D}$ & $\mathrm{FF}$ & 3 & 1 & & & & & \\
\hline
\end{tabular}




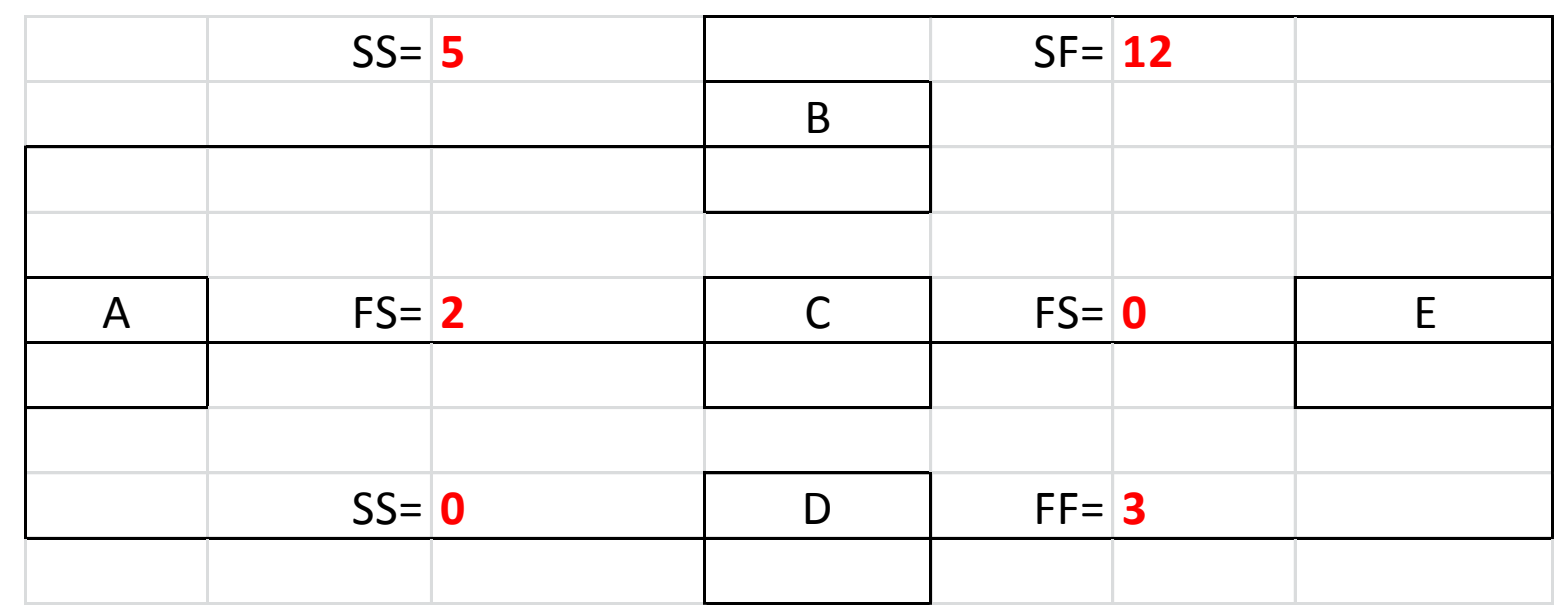

\section{Figure 3: Hypothetical Project Network}

\subsection{Solution Steps:}

1. Number of alternatives (Solutions) $=2 \times 2 \times 2 \times 2 \times 2=32$

2. Apply equations from (1 to 20) By using Excel spread sheets macro to Solve the first model

Table 2: Project Solutions (Alternatives) and corresponding Normal Duration and Cost

\begin{tabular}{|c|c|c|c|c|c|c|c|c|c|c|c|c|c|c|c|}
\hline \multirow{2}{*}{ Sol \# } & \multicolumn{5}{|c|}{ Solution Address } & \multicolumn{5}{|c|}{ Corrosponding Duration } & \multicolumn{5}{|c|}{ Corresponding Cost } \\
\hline & A & B & C & D & E & A & B & $\mathrm{C}$ & D & E & A & B & \begin{tabular}{|c|}
$\mathbf{C}$ \\
\end{tabular} & D & $\mathbf{E}$ \\
\hline 1 & 1 & 1 & 1 & 1 & 1 & 20 & 15 & 13 & 14 & 12 & 40000 & 450000 & 200000 & 400000 & 300000 \\
\hline 2 & 1 & 1 & 1 & 1 & 2 & 20 & 15 & 13 & 14 & 21 & 40000 & 450000 & 200000 & 400000 & 240000 \\
\hline 3 & 1 & 1 & 1 & 2 & 1 & 20 & 15 & 13 & 18 & 12 & 40000 & 450000 & 200000 & 320000 & 300000 \\
\hline 4 & 1 & 1 & 1 & 2 & 2 & 20 & 15 & 13 & 18 & 21 & 40000 & 450000 & 200000 & 320000 & 240000 \\
\hline 5 & 1 & 1 & 2 & 1 & 1 & 20 & 15 & 26 & 14 & 12 & 40000 & 450000 & 150000 & 400000 & 300000 \\
\hline 6 & 1 & 1 & 2 & 1 & 2 & 20 & 15 & 26 & 14 & 21 & 40000 & 450000 & 150000 & 400000 & 240000 \\
\hline 7 & 1 & 1 & 2 & 2 & 1 & 20 & 15 & 26 & 18 & 12 & 40000 & 450000 & 150000 & 320000 & 300000 \\
\hline 8 & 1 & 1 & 2 & 2 & 2 & 20 & 15 & 26 & 18 & 21 & 40000 & 450000 & 150000 & 320000 & 240000 \\
\hline 9 & 1 & 2 & 1 & 1 & 1 & 20 & 16 & 13 & 14 & 12 & 40000 & 350000 & 200000 & 400000 & 300000 \\
\hline 10 & 1 & 2 & 1 & 1 & 2 & 20 & 16 & 13 & 14 & 21 & 40000 & 350000 & 200000 & 400000 & 240000 \\
\hline 11 & 1 & 2 & 1 & 2 & 1 & 20 & 16 & 13 & 18 & 12 & 40000 & 350000 & 200000 & 320000 & 300000 \\
\hline 12 & 1 & 2 & 1 & 2 & 2 & 20 & 16 & 13 & 18 & 21 & 40000 & 350000 & 200000 & 320000 & 240000 \\
\hline 13 & 1 & 2 & 2 & 1 & 1 & 20 & 16 & 26 & 14 & 12 & 40000 & 350000 & 150000 & 400000 & 300000 \\
\hline 14 & 1 & 2 & 2 & 1 & 2 & 20 & 16 & 26 & 14 & 21 & 40000 & 350000 & 150000 & 400000 & 240000 \\
\hline 15 & 1 & 2 & 2 & 2 & 1 & 20 & 16 & 26 & 18 & 12 & 40000 & 350000 & 150000 & 320000 & 300000 \\
\hline 16 & 1 & 2 & 2 & 2 & 2 & 20 & 16 & 26 & 18 & 21 & 40000 & 350000 & 150000 & 320000 & 240000 \\
\hline 17 & 2 & 1 & 1 & 1 & 1 & 23 & 15 & 13 & 14 & 12 & 50000 & 450000 & 200000 & 400000 & 300000 \\
\hline 18 & 2 & 1 & 1 & 1 & 2 & 23 & 15 & 13 & 14 & 21 & 50000 & 450000 & 200000 & 400000 & 240000 \\
\hline 19 & 2 & 1 & 1 & 2 & 1 & 23 & 15 & 13 & 18 & 12 & 50000 & 450000 & 200000 & 320000 & 300000 \\
\hline 20 & 2 & 1 & 1 & 2 & 2 & 23 & 15 & 13 & 18 & 21 & 50000 & 450000 & 200000 & 320000 & 240000 \\
\hline 21 & 2 & 1 & 2 & 1 & 1 & 23 & 15 & 26 & 14 & 12 & 50000 & 450000 & 150000 & 400000 & 300000 \\
\hline 22 & 2 & 1 & 2 & 1 & 2 & 23 & 15 & 26 & 14 & 21 & 50000 & 450000 & 150000 & 400000 & 240000 \\
\hline 23 & 2 & 1 & 2 & 2 & 1 & 23 & 15 & 26 & 18 & 12 & 50000 & 450000 & 150000 & 320000 & 300000 \\
\hline 24 & 2 & 1 & 2 & 2 & 2 & 23 & 15 & 26 & 18 & 21 & 50000 & 450000 & 150000 & 320000 & 240000 \\
\hline 25 & 2 & 2 & 1 & 1 & 1 & 23 & 16 & 13 & 14 & 12 & 50000 & 350000 & 200000 & 400000 & 300000 \\
\hline 26 & 2 & 2 & 1 & 1 & 2 & 23 & 16 & 13 & 14 & 21 & 50000 & 350000 & 200000 & 400000 & 240000 \\
\hline 27 & 2 & 2 & 1 & 2 & 1 & 23 & 16 & 13 & 18 & 12 & 50000 & 350000 & 200000 & 320000 & 300000 \\
\hline 28 & 2 & 2 & 1 & 2 & 2 & 23 & 16 & 13 & 18 & 21 & 50000 & 350000 & 200000 & 320000 & 240000 \\
\hline 29 & 2 & 2 & 2 & 1 & 1 & 23 & 16 & 26 & 14 & 12 & 50000 & 350000 & 150000 & 400000 & 300000 \\
\hline 30 & 2 & 2 & 2 & 1 & 2 & 23 & 16 & 26 & 14 & 21 & 50000 & 350000 & 150000 & 400000 & 240000 \\
\hline 31 & 2 & 2 & 2 & 2 & 1 & 23 & 16 & 26 & 18 & 12 & 50000 & 350000 & 150000 & 320000 & 300000 \\
\hline 32 & 2 & 2 & 2 & 2 & 2 & 23 & 16 & 26 & 18 & 21 & 50000 & 350000 & 150000 & 320000 & 240000 \\
\hline
\end{tabular}




\begin{tabular}{|c|c|c|c|c|c|c|c|c|c|c|c|c|}
\hline & & & $\mathrm{FS}(\mathrm{m})=$ & -132 & & B & & $\mathrm{FS}(\mathrm{m})=$ & -112 & & & \\
\hline & & & & & & 160 & & & & & & \\
\hline \multirow[t]{2}{*}{0} & & 200 & & & 85 & & 215 & & & 138 & & 258 \\
\hline & $A$ & & & & & $C$ & & & & & $E$ & \\
\hline & 200 & & $\mathrm{FS}(\mathrm{m})=$ & -115 & & 130 & & $\mathrm{FS}(\mathrm{m})=$ & -97 & & 120 & \\
\hline & & & & & & & & & & & & \\
\hline & & & & & 72 & & 252 & & & & & \\
\hline & & & & & & D & & & & & & \\
\hline & & & $\mathrm{FS}(\mathrm{m})=$ & -128 & & 180 & & $\mathrm{FS}(\mathrm{m})=$ & -114 & & & \\
\hline & & & & & & & & & & & & \\
\hline
\end{tabular}

Figure 4: Optimum Project Normal Time \& corresponding Total Cost Table 3: Optimum Project Normal Duration and Cost

\begin{tabular}{|c|c|c|c|c|c|c|c|c|c|c|}
\hline Solution & A & B & C & D & E & $\begin{array}{c}\text { P.D } \\
\text { (days) }\end{array}$ & $\begin{array}{c}\text { P.C } \\
\text { (EGP) }\end{array}$ & $\mathrm{Z1}$ & $\mathbf{Z 2}$ & $0.5 \mathrm{Z1}+0.5 \mathrm{Z2}$ \\
\hline 1 & 1 & 1 & 1 & 1 & 1 & 246 & $1,463,800$ & -1.98 & -78.87 & -40.4244 \\
\hline 2 & 1 & 1 & 1 & 1 & 2 & 321 & $1,426,300$ & 0.48 & -79.39 & -39.4541 \\
\hline 3 & 1 & 1 & 1 & 2 & 1 & 258 & $1,387,400$ & -1.58 & -79.93 & -40.7568 \\
\hline 4 & 1 & 1 & 1 & 2 & 2 & 321 & $1,346,300$ & 0.48 & -80.50 & -40.0082 \\
\hline 5 & 1 & 1 & 2 & 1 & 1 & 294 & $1,428,200$ & -0.40 & -79.36 & -39.8837 \\
\hline 6 & 1 & 1 & 2 & 1 & 2 & 320 & $1,376,000$ & 0.45 & -80.09 & -39.8189 \\
\hline 7 & 1 & 1 & 2 & 2 & 1 & 294 & $1,348,200$ & -0.40 & -80.47 & -40.4379 \\
\hline 8 & 1 & 1 & 2 & 2 & 2 & 319 & $1,295,700$ & 0.42 & -81.20 & -40.3915 \\
\hline 9 & 1 & 2 & 1 & 1 & 1 & 246 & $1,363,800$ & -1.98 & -80.26 & -41.1171 \\
\hline 10 & 1 & 2 & 1 & 1 & 2 & 321 & $1,326,300$ & 0.48 & -80.78 & -40.1468 \\
\hline 11 & 1 & 2 & 1 & 2 & 1 & 258 & $1,287,400$ & -1.58 & -81.31 & -41.4495 \\
\hline 12 & 1 & 2 & 1 & 2 & 2 & 321 & $1,246,300$ & 0.48 & -81.88 & -40.7009 \\
\hline 13 & 1 & 2 & 2 & 1 & 1 & 294 & $1,328,200$ & -0.40 & -80.75 & -40.5764 \\
\hline 14 & 1 & 2 & 2 & 1 & 2 & 320 & $1,276,000$ & 0.45 & -81.47 & -40.5116 \\
\hline 15 & 1 & 2 & 2 & 2 & 1 & 294 & $1,248,200$ & -0.40 & -81.86 & -41.1306 \\
\hline 16 & 1 & 2 & 2 & 2 & 2 & 319 & $1,195,700$ & 0.42 & -82.58 & -41.0842 \\
\hline 17 & 2 & 1 & 1 & 1 & 1 & 273 & $1,481,900$ & -1.09 & -78.62 & -39.8562 \\
\hline 18 & 2 & 1 & 1 & 1 & 2 & 351 & $1,445,300$ & 1.47 & -79.13 & -38.8304 \\
\hline 19 & 2 & 1 & 1 & 2 & 1 & 285 & $1,405,500$ & -0.70 & -79.68 & -40.1886 \\
\hline 20 & 2 & 1 & 1 & 2 & 2 & 351 & $1,365,300$ & 1.47 & -80.23 & -39.3846 \\
\hline 21 & 2 & 1 & 2 & 1 & 1 & 297 & $1,439,100$ & -0.31 & -79.21 & -39.7590 \\
\hline 22 & 2 & 1 & 2 & 1 & 2 & 347 & $1,394,100$ & 1.33 & -79.84 & -39.2507 \\
\hline 23 & 2 & 1 & 2 & 2 & 1 & 297 & $1,359,100$ & -0.31 & -80.32 & -40.3132 \\
\hline 24 & 2 & 1 & 2 & 2 & 2 & 327 & $1,308,100$ & 0.68 & -81.03 & -40.1744 \\
\hline 25 & 2 & 2 & 1 & 1 & 1 & 273 & $1,381,900$ & -1.09 & -80.01 & -40.5489 \\
\hline 26 & 2 & 2 & 1 & 1 & 2 & 351 & $1,345,300$ & 1.47 & -80.51 & -39.5231 \\
\hline 27 & 2 & 2 & 1 & 2 & 1 & 285 & $1,305,500$ & -0.70 & -81.06 & -40.8813 \\
\hline 28 & 2 & 2 & 1 & 2 & 2 & 351 & $1,265,300$ & 1.47 & -81.62 & -40.0773 \\
\hline 29 & 2 & 2 & 2 & 1 & 1 & 297 & $1,339,100$ & -0.31 & -80.60 & -40.4517 \\
\hline 30 & 2 & 2 & 2 & 1 & 2 & 347 & $1,294,100$ & 1.33 & -81.22 & -39.9434 \\
\hline 31 & 2 & 2 & 2 & 2 & 1 & 297 & $1,259,100$ & -0.31 & -81.71 & -41.0059 \\
\hline \multirow[t]{5}{*}{32} & 2 & 2 & 2 & 2 & 2 & 327 & $1,208,100$ & 0.68 & -82.41 & -40.8671 \\
\hline & & & & & $\Sigma$ & 9802 & $42,940,600$ & & & \\
\hline & & & & & AVG & 306.313 & $7,156,767$ & & & \\
\hline & & & & & $\sum\left(x^{2}\right)$ & 3032214 & $57,788,447,260,000$ & & & \\
\hline & & & & & & 30.4851 & 72,181 & & & \\
\hline
\end{tabular}


The table (3) shows that:

1. Solution No. (11) is the optimum for min Project Normal Time \& corresponding Total Cost:

- PND (opt) $=258$ Days

- Direct Cost $=1,210,000 \mathrm{LE}$

- Indirect Cost $=77,400 \mathrm{LE}$

- Total Project Cost $=1,287,400$ LE

2. Apply Equation No. $(22,25)$ to calculate the Cost Slope and the Max Reduction Y as illustrated in table no. (4)

Table 4: Cost Slope and Max Reduction Units of Project Activities

\begin{tabular}{||c|c|c|c|c|c|c||}
\hline Act & NT & CT & NC & CC & $\max Y$ & CS \\
\hline \hline A & 200 & 170 & 50000 & 58000 & 30 & 266.667 \\
\hline B & 160 & 140 & 350000 & 371000 & 20 & 1050 \\
\hline C & 130 & 120 & 150000 & 154000 & 10 & 400 \\
\hline D & 180 & 160 & 320000 & 325000 & 20 & 250 \\
\hline E & 120 & 100 & 240000 & 264000 & 20 & 1200 \\
\hline
\end{tabular}

\subsection{Decision Variables:}

$\mathrm{X} 1=\mathrm{Y}_{\mathrm{A}}, \mathrm{X} 2=\mathrm{Y}_{\mathrm{B}}, \mathrm{X} 3=\mathrm{Y}_{\mathrm{C}}, \mathrm{X} 4=\mathrm{Y}_{\mathrm{D}}, \mathrm{X} 5=\mathrm{Y}_{\mathrm{E}}, \mathrm{X} 6=\mathrm{PD}, \mathrm{X} 7=\mathrm{S}_{\mathrm{B}}, \mathrm{X} 8=\mathrm{S}_{\mathrm{C}}, \mathrm{X} 9=\mathrm{S}_{\mathrm{D}}, \mathrm{X} 10=\mathrm{S}_{\mathrm{E}}$

\subsection{Objective Function}

$Z \min =266.667 \times Y_{A}+1050 \times Y_{B}+400 \times Y_{C}+250 \times Y_{D}+1200 \times Y_{E}$

\subsection{Constraints:}

$$
+300 \times P D_{c}
$$

\subsubsection{Nonnegative constraints:}

$\mathrm{Y}_{\mathrm{A}}, \mathrm{Y}_{\mathrm{B}}, \mathrm{Y}_{\mathrm{C}}, \mathrm{Y}_{\mathrm{D}}, \mathrm{Y}_{\mathrm{E}}, \mathrm{S}_{\mathrm{B}}, \mathrm{S}_{\mathrm{C}}, \mathrm{S}_{\mathrm{D}}, \mathrm{S}_{\mathrm{E}}, \mathrm{PD} \geq 0$

\subsubsection{Maximum reduction constraints:}

$$
\begin{aligned}
& Y_{A} \leq 30 \\
& Y_{B} \leq 20 \\
& Y_{C} \leq 10 \\
& Y_{D} \leq 20 \\
& Y_{E} \leq 20
\end{aligned}
$$

\subsection{3. $\quad$ Start Time Constraints:}

$$
\begin{aligned}
& S_{B} \geq S_{A}+S \times N D_{A}-Y_{A}+\left(F S_{m}\right)_{A, B} \\
& S_{A}=0 \\
& S_{B}+Y_{A} \geq 68 \\
& S_{C}+Y_{A} \geq 85 \\
& S_{D}+Y_{A} \geq 72
\end{aligned}
$$

$$
S_{E} \geq S_{B-} Y_{A}+1160-112
$$


$S_{E}-S_{B}+Y_{B} \geq 48$
$S_{E}-S_{C}+Y_{C} \geq 33$
$S_{E}-S_{D}+Y_{D} \geq 66$

\subsubsection{Project duration constraints:}

$P D_{c} \geq S_{E}+N D_{E} \times S-Y_{E}$

So; $P D_{c}-S_{E}+Y_{E} \geq 120$

$P D_{c} \leq 258$

- By using Lips Software to solve the model the and Comparing the data with Excel Solver Results following results has been found:

Table 5: Lips Software Results

\begin{tabular}{|c|c|c|c|}
\hline Variable & Value & Obj. Cost & Reduced Cost \\
\hline \hline X1 & 30 & 266.667 & 0 \\
\hline X2 & 0 & 1050 & -1050 \\
\hline X3 & 0 & 400 & -400 \\
\hline X4 & 20 & 250 & 0 \\
\hline X5 & 0 & 1200 & 0 \\
\hline X6 & 208 & 300 & 0 \\
\hline X7 & 40 & 0 & 0 \\
\hline X8 & 55 & 0 & 0 \\
\hline X9 & 42 & 0 & 0 \\
\hline X10 & 88 & 0 & \\
\hline \hline
\end{tabular}


Table 6: Excel Solver Results

\begin{tabular}{|r|c|c|c|c|c|c|c|c|c|c|c|c|}
\hline \multicolumn{2}{|l|}{ SOLVER } \\
\hline
\end{tabular}

\section{Project Cost (EGP)}

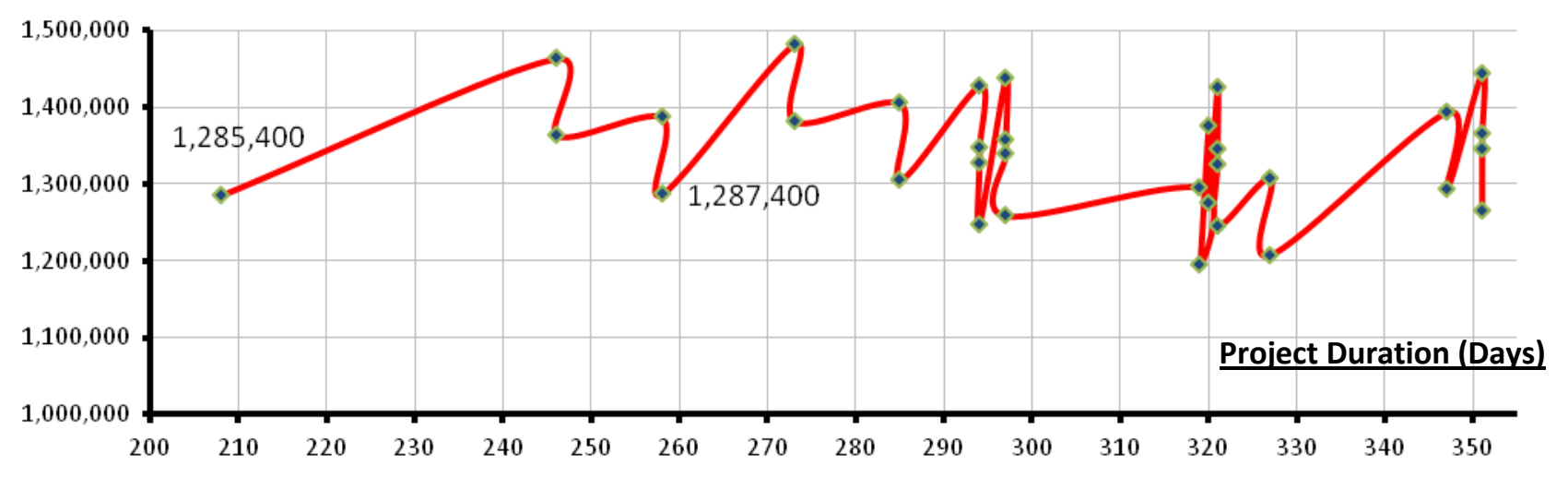

Figure 5: Comparing Model Result (Crashed) with The Normal Values (32 Solutions)

\section{Analysis and Results}

The linear programming model will not only take into account the activities on the critical path, but will also consider the non-critical activities, which in their turn become critical as the project time decreases.

Using LiPS software and Excel Solver; the solution of the model is presented in table(5) and (6), which indicates that:

1. The Optimum Min Project TC $=1,210,000+75,400=(\mathbf{1 , 2 8 5}, \mathbf{4 0 0 E G P})$ corresponding to (208)

Day Project Duration.

2. These results are due to crashing Activity (A) (3 days per stage) and Activity (D) (2 Days per stage).

It requires trial and error method to get the optimal result. It is important for project manager to recognize the flexibility of the system that can be used to explore numerous possible opportunities to the contractor. Moreover, this approach allows the user to easily manipulate different project 
networks of various difficulties representing real world applications, and to study the effectiveness of the model in the case of large projects.

The implementation of the developed model showed more efficient and reliable results and generated a considerable computational savings along with an increase in robustness.

\section{Conclusion}

The data needed for crashing project activities by means of linear programming technique are the time and cost for each activity when it is done in the normal way and then when it is fully crashed (expedited). The project manager can investigate the effect on total cost of changing the estimated duration of the project to various alternative values. Using linear programming model, the project manager will be able to determine how much (if any) to crash each activity in order to minimize the total cost of the project.

An algorithmic model based on linear programming incorporated with a minimal time-cost crash in a construction project was introduced. The format of the model lends itself to a wide range of variables and considerations.

The introduced modeling strategy which showed the resources of this interactive approach including a bulk of data to completely analyze the project is easily possible. It allowed a great number of parameters to simulate project conditions and contractor's preference and provided potentially useful tool for decision making on project scheduling.

\section{References}

1. Hafez S. M. (1997a). "Resource Allocation and Smoothening for Repetitive Projects" M. Sc. Thesis, Structural Eng. Dept., Alex. Univ., Alex., Egypt.

2. Hafez S. M. (2005). "Developing Classical Schedule Technology" Alexandria Engineering Journal, Vol. 44 (2), pp. C267-C277.

3. Selinger S., "Construction planning for linear projects", ASCE Journal of the Construction Division, Vol. 106, 2, (1980), pp.195-205.

4. Russel A.D. and Caselton W.F., "Extensions to linear scheduling optimization", ASCE Journal of Construction Engineering and Management, Vol.114, 1, (1988), pp.36-52.

5. Reda R.M., "RPM: repetitive project modeling", ASCE Journal of Construction Engineering and Management, 116, 2, (1990), pp. 316-330.

6. Elmaghraby S.E. and Pulat P.S., "Optimal Project Compression with Due-Dated Events, "Naval Research Logistics Quarterly, Vol.26, º2, (1997), pp. 331-348.

7. Moore L.J., Taylor III B.W., Clayton E.R., and Lee S.M., "Analysis of a Multi-Criteria Project Crashing Model," American Institute of Industrial Engineering Trams., Vol. 10, №2, (1998), pp. 163-169.

8. Senouci A.B. and Eldin N.N., "A time-cost trade-off algorithm for non-serial linear projects", Canadian J. of Engineering, Vol.23, (1996), pp.134-149.

9. Remon Fayek Aziz, 2010, "Time-Cost-Quality-Trade- Off for construction project with repetitive activities".

10. Smith L.A., "Comparing Commercially Available CPM/PERT Computer Programs", Journal of Industrial Engineering, Vol. 10, $\mathrm{N}^{\circ} 4,(1997)$.

11. Schrage L.E., User's manual for LINDO; Scientific Press, Palo Alto, California, (1981).

12. Al Serraj Z.M., "Formal development of line-of balance technique", ASCE Journal of Construction Engineering and Management, Vol.116, 4, (1990), pp. 689-704.

13. Siemens N., "A Simple CPM Time-Cost Trade-off Algorithm," Management Science, Vol.17, No. 6, 1997), pp.B354-363.

14. Abo Elmagd Y. M. (2005). "Time-Cost Trade-Off in Construction Projects Applied on a Single Tower Crane" M. Sc. Thesis, Structural Eng. Dept., Alex. Univ., Alex., Egypt. 
15. Adeli H. and Karim A. (1997). "Scheduling/Cost Optimization and Neural Dynamics Model for Construction" J. Constr. Engrg. and Mgmt., ASCE, 123 (4), 450-458.

16. Ahsan K., and Gunawan I. (2010). "Analysis of Cost and Schedule Performance of International Development Projects" International Journal of Project Management, (28), 68-78.

17. Chassiakos A. B. and Sakellaropoulos P.S. (2005). "Time-Cost Optimization of Construction Projects with Generalized Activity Constraints" J. Constr. Eng. And Mgmt., ASCE, 131 (10), 1115-1124. 